\title{
Apparently diffuse epileptic abnormalities caused by a small cavernous malformation: a surgical case report
}

Atsuhiko Ninomiya ${ }^{1}$, Masaki Iwasaki ${ }^{1,2}$, Yosuke Kakisaka ${ }^{3}$, Kazutaka $\mathrm{Jin}^{3}$, Nobukazu Nakasato ${ }^{3}$, Teiji Tominaga $^{1}$

${ }^{1}$ Department of Neurosurgery, Tohoku University Graduate School of Medicine, Sendai, Miyagi, Japan

${ }^{2}$ Department of Neurosurgery, National Center Hospital, National Center of Neurology and Psychiatry, Kodaira, Tokyo, Japan

${ }^{3}$ Department of Epileptology, Tohoku University Graduate School of Medicine, Sendai, Miyagi, Japan

Key words: Cavernous malformation; Epilepsy surgery; Seizure outcome; Pre-surgical evaluation; EEG

Received: May 3, 2018; Accepted: December 3, 2018

\begin{abstract}
Resective surgery is considered if the lesion location is concordant with the epileptogenic zone evaluated by electrophysiological assessment. Diffuse epileptic abnormalities may lead to extended resection or preclude an indication for surgical treatment. We report a 17-year-old boy with a small cavernous malformation presenting drug-resistant epilepsy. Although pre-surgical evaluations revealed epileptic abnormalities widely around the lesion, and invasive evaluation with implanted subdural electrodes failed to localize seizure onset to the lesion, his seizures were significantly improved after simple lesionectomy. A small cavernous malformation could cause apparently diffuse functional abnormalities, and simple lesionectomy may be a reasonable option for drug-resistant epilepsy associated with a cavernous malformation.
\end{abstract}




\section{Introduction}

Cavernous malformation (CM) is a major cause of drug-resistant epilepsy. Preoperative estimation of the epileptogenic zone with techniques such as MRI, long-term video EEG (VEEG), and magnetoencephalography (MEG) are very important for planning epilepsy surgery [1]. If the epileptic zone is concordant with the location of the CM, simple lesionectomy including the $\mathrm{CM}$ and surrounding cortex with hemosiderin deposition is recommended for seizure control [2]. However, if epileptic activities are observed extending widely around the lesion, surgical removal may be precluded due to the low probability of seizure control and functional preservation, as well as the surgical risks.

We report a surgical case of drug-resistant epilepsy associated with a small CM located in the right middle frontal gyrus. Presurgical evaluations revealed diffuse epileptic abnormalities, and chronic intracranial EEG failed to show focal abnormalities associated with the lesion, but the seizures were successfully controlled after simple lesionectomy.

\section{Case presentation}

All procedures in this study followed the clinical study guidelines of Tohoku University Hospital and were approved by the ethics committee. The patient and his caregivers provided written informed consent.

A 17-year-old male with unremarkable family and past medical history had onset of weekly seizures from the age of 10 years. His seizures were characterized by sudden palpitation and contraction of his right hand for 10 seconds without loss of consciousness, but sometimes progressed to secondarily general- ized tonic-clonic seizures (sGTC) with extension of the right arm and rotation of the body to the left for one minute. The simple partial seizures occurred once a week and sGTC occurred monthly, despite multiple antiepileptic medications including valproate, levetiracetam, carbamazepine, zonisamide, and clonazepam. He was taking daily doses of $400 \mathrm{mg}$ valproate and $750 \mathrm{mg}$ levetiracetam when referred to us for surgical treatment evaluation.

Comprehensive presurgical evaluations of epilepsy were carried out. Two electroclinical seizures were recorded on VEEG. The seizure started with sudden palpitation and loss of sensation in the left upper arm, followed by flexion and elevation of the right arm, rotation of the body to the left, and then secondarily generalized tonic-clonic convulsion. Ictal EEG was characterized by diffuse irregular theta-delta rhythms intermixed with spikes, which were interpreted as nonlocalizable onset (Fig. 1A). Diffuse interictal high-amplitude spikes were detected at maximum amplitude in the right frontal region.

Axial T2-weighted MRI showed a hypointense lesion in the right middle frontal gyrus just anterior to the precentral sulcus (Fig. 2A), suggestive of CM. MEG showed equivalent current dipoles of the interictal spikes distributed widely around the lesion (Fig. 2B). Interictal fluorodeoxyglucose positron emission tomography (FDG-PET) showed focal glucose hypometabolism at the lesion and relatively diffuse hypometabolism in the right anterior frontal lobe (Fig. 2C). Neuropsychologically, his performance intelligence quotient was relatively low, but otherwise unremarkable. 

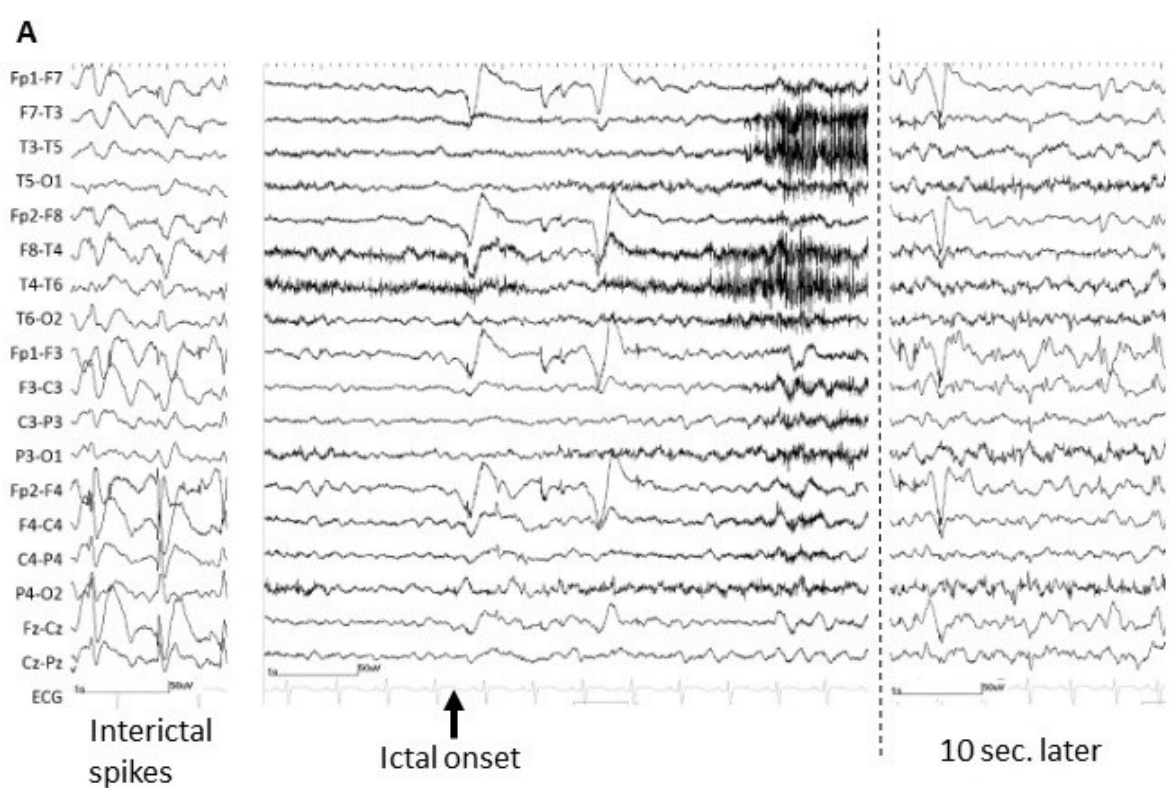

B

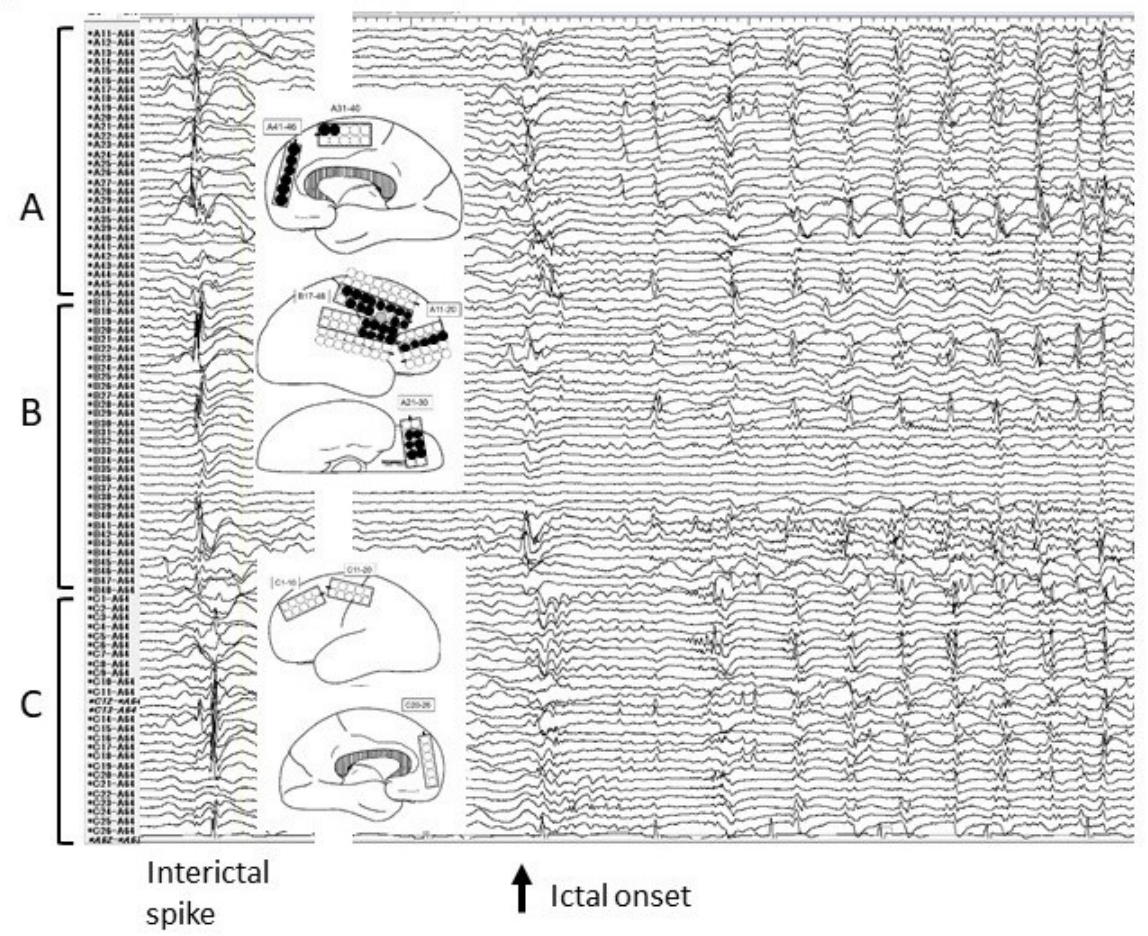

Figure 1. (A) Scalp EEG findings. Interictal diffuse high-amplitude spikes are seen with maximum amplitude in the right frontal region (left). The EEG seizure starts with isolated spikes in the right frontal region (middle), but diffuse and slow rhythmic change rapidly follows, associated with spikes in the left hemisphere (right). (B) Intracranial EEG findings. Cortical electrode A was placed on the anterior lateral, basal, and medial surface of the right frontal lobe. Cortical electrode B was placed on the right lateral frontal lobe around the lesion. Cortical electrode $\mathrm{C}$ was placed on the left medial and lateral frontal lobe. Interictal spikes are distributed diffusely over both right and left frontal cortices, poorly localized in the lesion (left). Ictal EEG starts with a diffuse spike in the right frontal lobe marked as black dots, followed immediately by repetitive spikes in the bilateral frontal lobes considered as mirror focus described by Jin et al [6] (right). 

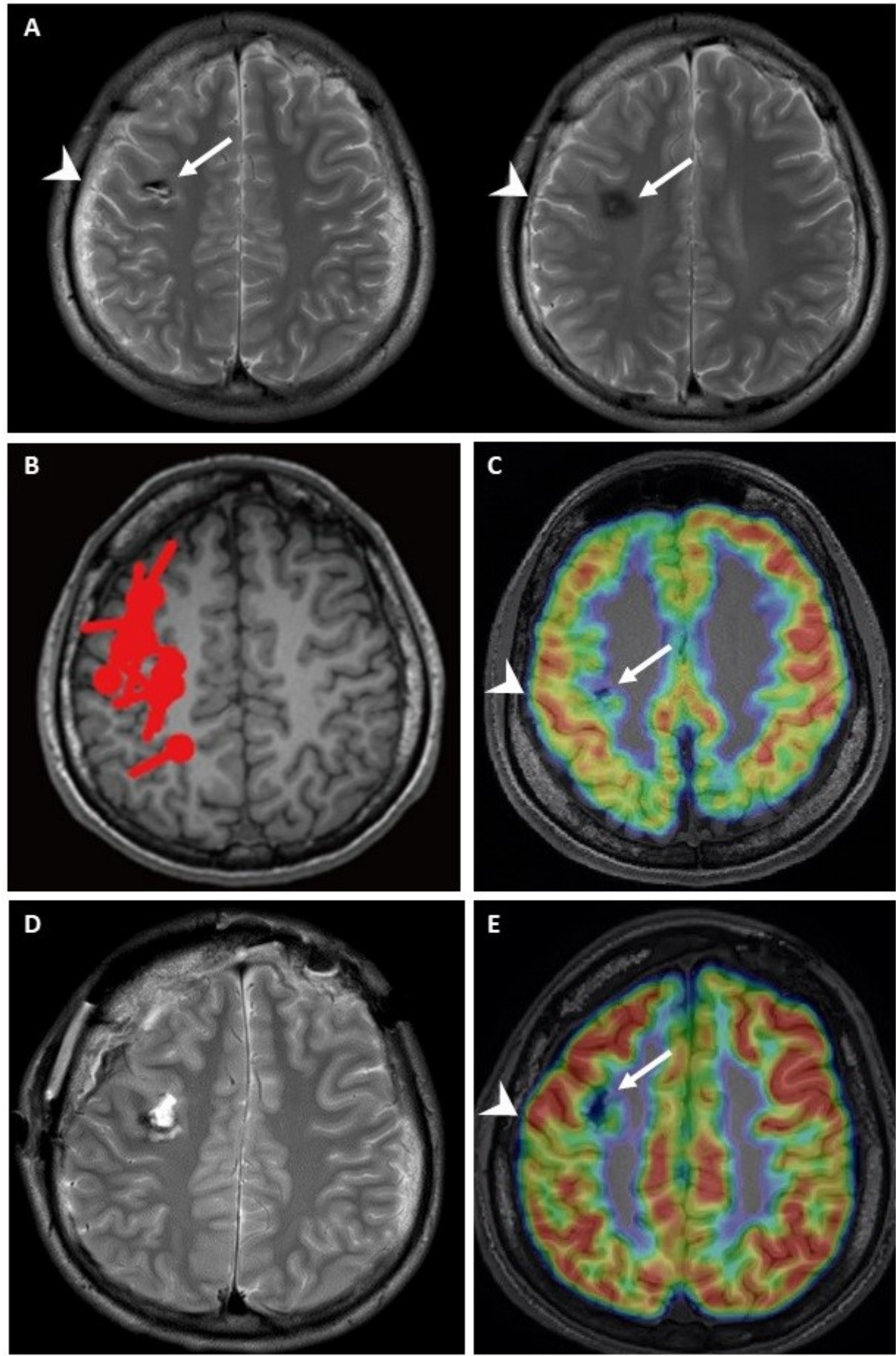

Figure 2. (A) Preoperative axial T2-weighted MRI showing a hypointense lesion (arrow) in the right middle frontal gyrus just anterior to the precentral sulcus. The central sulcus is indicated by the arrowhead. (B) MEG spike mapping showing equivalent current dipoles of interictal spikes distributed widely around the lesion. (C) Preoperative interictal FDG-PET scan showing focal glucose hypometabolism at the lesion and relatively diffuse hypometabolism in the right anterior frontal lobe. (D) Postoperative T2-weighted MRI showing the state after removal of the CM. (E) FDG-PET scan at one year after surgery showing improvement of glucose hypometabolism in the right frontal lobe. 
Invasive VEEG with chronically implanted intracranial electrodes was performed for two weeks, because the non-invasive evaluations suggested wider epileptogenic zone than the lesion. A total of 110 and 26 subdural electrodes were implanted in the right and left frontal lobes, respectively. Five electroclinical seizures were recorded during the VEEG monitoring. Electrocorticography indicated diffusely distributed interictal spikes and poorly localized ictal EEG changes (Fig. 1B). Therefore, we concluded that the epileptogenic zone was not localized to the $\mathrm{CM}$ or even to the frontal lobe covered by the electrodes. Simple lesionectomy was planned to minimize the risk of future hemorrhagic complications, with expectation of a fair chance of seizure control.

Surgery was performed under general anesthesia with motor-evoked potential monitoring of the left adductor pollicis muscle. The precentral sulcus was sharply dissected before the hemosiderin deposited cortex was identified. The berry-like lesion was completely removed. Removal of the surrounding cortex containing hemosiderin deposits was performed by suction and bipolar cautery, but the pre-central gyrus was preserved to minimize postoperative neurological complication. Postoperatively, total removal of the CM was confirmed (Fig. 2D), and no neurological complication was observed.

He was followed and treated with the same antiepileptic medications. A single unprovoked generalized tonic-clonic seizure occurred at eight months after surgery, and the dose of levetiracetam was increased to 1000 mg per day. Postoperative FDG-PET examination performed at one year confirmed normalized glucose metabolism (Fig. 2E) and
EEG showed absence of interictal spikes. Neuropsychological examination at one year after surgery showed no significant changes in intelligence quotient and other intellectual and memory performance. A single episode of brief partial seizure occurred approximately two years after surgery (ILAE class 3 ).

\section{Discussion}

Simple lesionectomy may be the first treatment option for drug-resistant epilepsy associated with $\mathrm{CM}$, regardless of the extent of epileptic activities in pre-surgical evaluation $[3,4,5]$. In the present case, the CM and the adjacent cortex were epileptogenic. Although preoperative investigations showed diffuse epileptic abnormalities, simple lesionectomy was sufficient to control his seizures with continuing antiepileptic medication.

Hemosiderin deposits around the CM trigger toxic excitability of neurons [2], leading to irritability of the adjacent cortex. The irritable cortex can induce epileptic activities that spread to contralateral homologous site as well as ipsilateral remote sites [6]. Such brain regions are thought to have kindling epileptogenicity via anatomo-functionally connections [4]. Bilateral synchronous discharges have been reported to be characteristic, but not specific, especially in frontal lobe epilepsy [7]. In our case, epileptiform activities were observed widely around the lesion, even in the opposite frontal lobe, suggesting rapid and extensive spread of the epileptic discharges originating around the frontal lesion.

Diffuse glucose hypometabolism may occur secondary to small CM. Few cases of CM 
-related epilepsy demonstrated perilesional hypometabolism. No apparent relationship was found between the epileptogenic nature of the CM and PET hypometabolism. The patients became seizure-free after lesionectomy, suggesting that the hypometabolism was caused by deafferentation rather than epileptogenicity [8]. In contrast, glucose hypometabolism in PET indicates the presence of epileptogenic pathology in focal cortical dysplasia [9]. In our case, diffuse glucose hypometabolism was normalized after lesionectomy, accompanied by amelioration of seizures and EEG abnormalities [10]. Therefore, removal of the hypometabolic areas surrounding the CM may be unnecessary [11].

In the present case, additional depth electrode recording may have revealed focal seizure onset from the vicinity of CM. Subdural electrodes are sometimes insensitive to deeply-located epileptic activities, such as those in the bottom of sulcus $[12,13]$. However, we believe that all EEG spikes were not a result of volume conduction of deep sources in this case. Rather, interictal epileptic activities and functional deficits may occur remote from the small epileptogenic lesion. Residual interictal epileptiform discharges after surgery are known to be a predictive factor of postoperative seizure outcome [10]. The clinical significance of remote interictal abnormalities should be further investigated.

\section{Acknowledgement}

We express sincere thanks to Dr. Shiho Sato and staff of the Department of Radiology, Tohoku University Hospital for figure editing of MRI-PET fusion images. This research was partially supported by Grants-in-
Aid for Scientific Research (KAKENHI) JP16K10780 and JP16H05435 from the Japan Society for the Promotion of Science.

\section{Conflicts of interest}

None of the authors have any conflict of interest to declare.

\section{References}

[1] Stefan H, Scheler G, Hummel C, Walter J, Romstock J, Buchfelder M, Blumcke I. Magnetoencephalography (MEG) predicts focal epileptogenicity in cavernomas. J Neurol Neurosurg Psychiatry 2004; 75: 1309-1313.

[2] Stefan H, Hammen T. Cavernous hemangiomas, epilepsy and treatment strategies. Acta Neurol Scand 2004; 110: 393397.

[3] Komotar RJ. Mikell CB, McKhann GM $2^{\text {nd }}$. "Epilepsy surgery" versus lesionectomy in patients with seizures secondary to cavernous malformations. Clinical Neurosurgery 2008; 55: 101-107.

[4] Jehi LE, Palmini A, Aryal U, Coras R, Paglioli E. Cerebral cavernous malformations in the setting of focal epilepsies: pathological findings, clinical characteristics, and surgical treatment principals. Acta Neuropathol 2014; 128: 55-65.

[5] Rathore C, Radhakrishnan K. Concept of epilepsy surgery and presurgical evaluation. Epileptic Disord 2015; 17: 19-31.

[6] Jin K, Nakasato N, Shamoto H, Kanno A, Itoyama $\mathrm{Y}$, Tominaga $\mathrm{T}$ : Neuromagnetic localization of spike sources in perilesional, contralateral mirror, and ipsilateral remote areas in patients with cavernoma. Epilepsia 2007; 48: 2160-2166. 
[7] Beleza P, Pinho J: Frontal lobe epilepsy. J Clin Neurosci 2011; 18: 593-600.

[8] Ryvlin P, Mauguière F, Shindou M, Froment JC, Cinotti L. Interictal cerebral metabolism and epilepsy in cavernous angiomas. Brain 1995; 118: 677-687.

[9] Lee SK, Kim DW. Focal cortical dysplasia and epilepsy surgery. J Epilepsy Res 2013; 3: 43-47.

[10]Elsharkawy A, Alabbasi A, Pannek H, Schulz R, Hoppe M, Pahs G, Nayel M, Issa A, Ebner A. Outcome of frontal lobe epilepsy surgery in adults. Epilepsy Res 2008; 81: 97-106.

[11]Stanišić M, Coello C, Ivanović J, Egge A, Danfors T, Hald J, Heminghyt E, Mikkelsen M, Krossnes B, Pripp A, Larsson P. Seizure outcomes in relation to the extent of resection of the perifocal fluorodeoxyglucose and flumazenil PET abnormalities in anteromedial temporal lobectomy. Acta Neurochir 2015; 157: 1905-1916.

[12] Vadera S, Mullin J, Bulacio J, Najm I, Bingaman W, Gonzalez-Martinez J: Stereoelectroencephalography following subdural grid placement for difficult to localize epilepsy. Neurosurgery 2013; 72: 723-729.

[13] Gonzalez-Martinez J, Bulacio J, Alexopoulos A, Jehi L, Bingaman W, Najm I. Stereoelectroencephalography in the 'difficult to localize' refractory focal epilepsy: Early experience from a North American epilepsy center. Epilepsia 2013; 54: 323-330. 